\title{
In the midst of a dangerous intersection with unclear therapeutic strategies: a challenging case of severe aortic stenosis
}

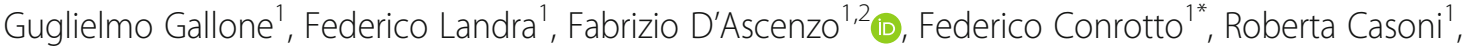 \\ Francesco Bruno ${ }^{1}$, Pierluigi Omedè ${ }^{1}$, Gianluca Alunni ${ }^{1}$, Alessandro Andreis ${ }^{1}$, Alessandro Vairo ${ }^{1}$, Mauro Giorgi ${ }^{1}$, \\ Antonella Fava ${ }^{1}$ and Gaetano Maria De Ferrari ${ }^{1}$
}

\begin{abstract}
Background: One out of seven patients with severe aortic stenosis (AS) undergoing transcatheter aortic valve replacement (TAVR) may be affected by transthyretin cardiac amyloidosis (ATTR-CA), mostly presenting with lowflow low-gradient AS with mildly reduced ejection fraction. The complex interaction of these two pathologies poses specific diagnostic and management challenges. The prognostic implications of this clinical intersection are not defined yet. Moreover, whether TAVR may have a prognostic benefit in ATTR-CA patients with symptomatic severe AS remains unclear, posing doubts on the best management strategy in this increasingly recognized subset of patients.

Clinical case: We present a case of an 87-year old man with low-flow low-gradient severe AS, for whom a diagnosis of ATTR-CA was suspected based on clinical and echocardiographic criteria specific to coexisting AS and ATTR-CA. The diagnosis was eventually confirmed by positive bone tracer scintigraphy imaging. Following in-depth Heart team discussion, integrating frailty and prognostic information from combined cardiomyopathy states, a decision was made to manage the patient's severe AS conservatively.

Conclusion: In the presented case, we deemed the natural history of ATTR-CA amyloidosis to negatively affect both the patient' prognosis and procedural risk, adversing TAVR indication despite symptomatic severe AS. No clear evidence is currently available to guide decision making in this setting, advocating for prospective studies to clarify if TAVR may have a prognostic benefit in ATTR-CA - and which ATTR-CA - patients.
\end{abstract}

Keywords: Aortic stenosis, Cardiac amyloidosis, Transcatheter aortic valve intervention, Strain, Bone tracer scintigraphy, Heart team, Case report

\footnotetext{
* Correspondence: fabrizio.dascenzo@gmail.com

'Department of Medical Sciences, University of Turin, Città della Salute e della Scienza Hospital, Turin, Italy

Full list of author information is available at the end of the article
}

(c) The Author(s). 2020 Open Access This article is licensed under a Creative Commons Attribution 4.0 International License, which permits use, sharing, adaptation, distribution and reproduction in any medium or format, as long as you give appropriate credit to the original author(s) and the source, provide a link to the Creative Commons licence, and indicate if changes were made. The images or other third party material in this article are included in the article's Creative Commons licence, unless indicated otherwise in a credit line to the material. If material is not included in the article's Creative Commons licence and your intended use is not permitted by statutory regulation or exceeds the permitted use, you will need to obtain permission directly from the copyright holder. To view a copy of this licence, visit http://creativecommons.org/licenses/by/4.0/ The Creative Commons Public Domain Dedication waiver (http://creativecommons.org/publicdomain/zero/1.0/) applies to the data made available in this article, unless otherwise stated in a credit line to the data. 


\section{Background}

One out of seven patients with severe aortic stenosis (AS) undergoing transcatheter aortic valve replacement (TAVR) may be affected by transthyretin cardiac amyloidosis (ATTR-CA), mostly presenting with low-flow low-gradient AS with mildly reduced ejection fraction [1]. The complex interaction of these two pathologies poses specific diagnostic and management challenges. The prognostic implications of this clinical intersection are not defined yet. Moreover, whether TAVR may have a prognostic benefit in ATTR-CA patients with symptomatic severe AS remains unclear, posing doubts on the best management strategy in this increasingly recognized subset of patients.

\section{Case presentation}

A 87-year old man with a presenting diagnosis of hypertensive cardiomyopathy, orthostatic hypotension, moderate aortic stenosis, permanent atrial fibrillation, left bundle branch block and preserved ejection fraction (EF) (home therapy: valsartan $80 \mathrm{mg}$, bisoprolol $2.5 \mathrm{mg}$ ) was admitted to our Clinic with Canadian Cardiovascular Society III angina, New York Heart Association (NYHA) III functional class and signs of pulmonary and systemic venous congestion (Fig. 1).

Cardiac ultrasound documented left ventricle (LV) hypertrophy (septum $16 \mathrm{~mm}$, posterior wall $15 \mathrm{~mm}, \mathrm{LV}$ mass index $189 \mathrm{~g} / \mathrm{m}^{2}$ ) with severe concentric remodeling (relative wall thickness 0.6$)$, mildly reduced EF (50\%), severely depressed global longitudinal strain (-3.1\%) with no relative apical sparing pattern and an average mitral annular Tissue Doppler $\mathrm{S}^{\prime}$ of $5.5 \mathrm{~cm} / \mathrm{sec}$. Second degree diastolic disfunction and severe biatrial dilatation were also apparent. A low QRS voltage-to-LV mass ratio by Sokolow-Lyon criteria was observed. Aortic valve evaluation revealed low flow-low gradient severe aortic stenosis (AS) (Vmax $2.6 \mathrm{~m} / \mathrm{sec}$, max/mean gradient $28 / 16 \mathrm{mmHg}$, indexed aortic valve functional area $0.4 \mathrm{~cm}^{2} / \mathrm{m}^{2}$, stroke volume index $\left.21 \mathrm{ml} / \mathrm{m}^{2}\right)$ ((Fig. 2), confirmed by computed tomography scan (aortic valve calcium score 2500).

Following successful decongestion and clinical improvement with intravenous diuretics and vasodilators,

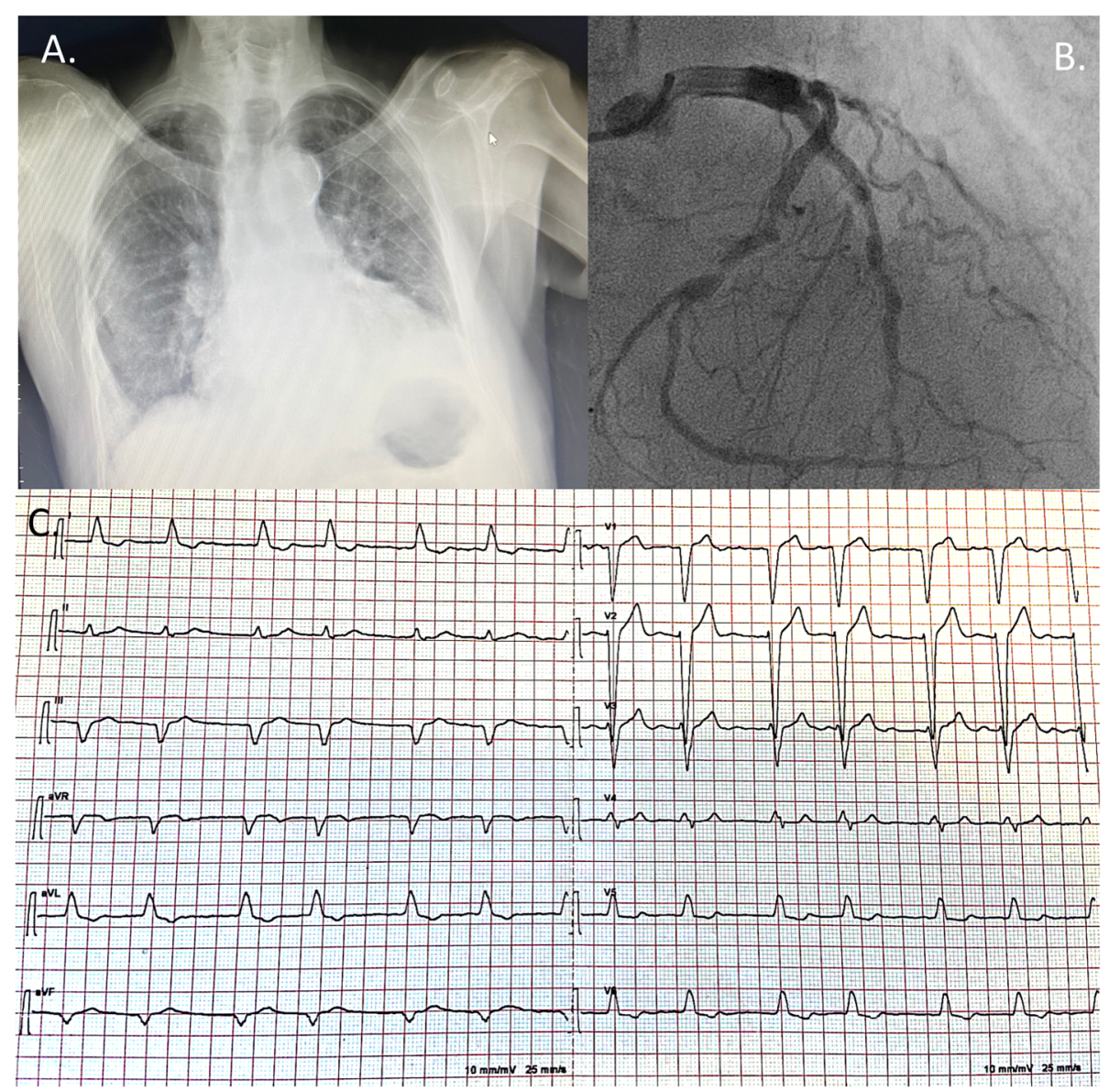

Fig. 1 a Chest x-ray at patient hospital admission showing lung congestion and enlarged heart shadow (top left). b Coronary angiography showing diffuse disease with critical disease of the mid and distal left anterior descending coronary artery and of the proximal and distal circumflex coronary artery (top right). c Electrocardiogram showing atrial fibrillation and left bundle branch block (bottom) 


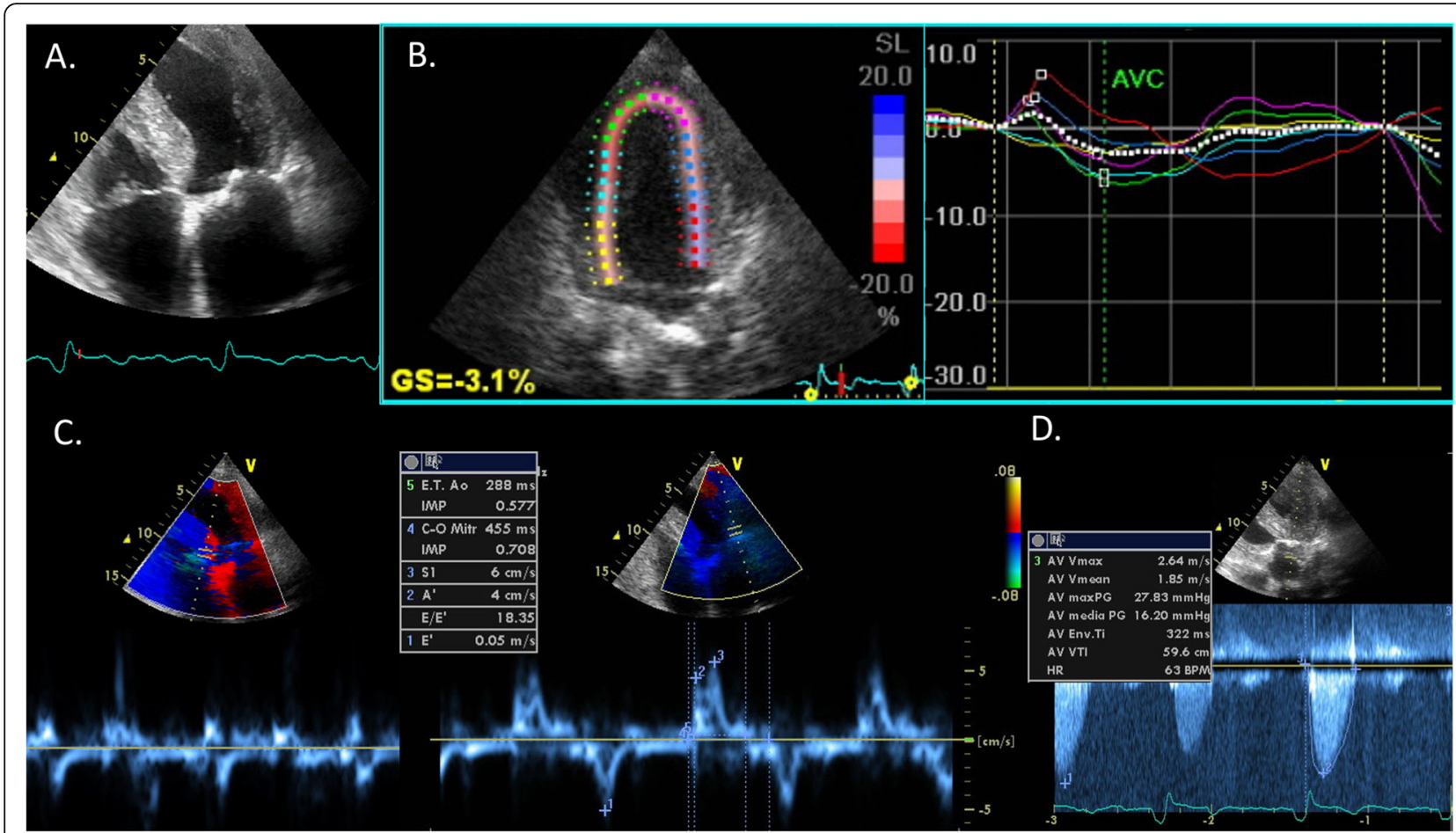

Fig. 2 a Transthoracic echocardiographic apical 4-chamber view showing severe LV hypertrophy with myocardial granular sparkling, biatrial dilatation and atrial septal thickening. $\mathbf{b}$ Longitudinal strain analysis revealing severely impaired longitudinal systolic function without apical sparing. c Lateral and septal mitral annular tissue Doppler showing a severely reduced average S'. d Continuous wave doppler across LV outflow tract showing low gradient AS

coronary angiography revealed critical multivessel diffuse disease, which was treated with complete percutaneous revascularization (two drug-eluting stents on the left anterior descending and circumflex coronary arteries).

Based on the low-flow low-gradient AS phenotype in the background of severe concentric hypertrophy, biatrial dilatation, $\mathrm{S}^{\prime}$ wave depression, conduction abnormalities, low QRS-voltage-to-LV-mass ratio and history of orthostatic hypotension, a diagnosis of amyloid cardiomyopathy was suspected. Bone tracer scintigraphy was performed revealing high diffuse myocardial uptake (Perugini score 3), diagnostic - in the context of an absent monoclonal protein on serum and urine exams (the patient had negative serum and urine immunofixation, and negative serum free light chain assay) - for transthyretin cardiac amyloidosis (ATTR-CA) [2] (Fig. 3).

The patient was unsuitable for ATTR-CA specific treatment because of current national reimbursement policies.

A Heart Team evaluation was carried. In consideration of the high procedural risk (EuroSCORE II 11.4\%) transcatheter aortic valve replacement (TAVR) appeared indicated for severe AS treatment. However, considering the patient frailty (Essential Frailty Toolset score of 4/5: preprocedural anemia (1 point), hypoalbuminemia (1 point), lower-extremity muscle weakness (2 points) [3]), the presence of ATTR-CA cardiomyopathy-specific markers of adverse prognosis (Mayo Clinic ATTR-CA stage III: high-sensitivity troponin $\mathrm{T}=280 \mathrm{ng} / \mathrm{L}, \quad \mathrm{NTproBNP}=$ $6500 \mathrm{ng} / \mathrm{L}$ [4]; echocardiographic parameters heralding poor prognosis in ATTR-CA with severe AS: reduced stroke volume, severely reduced global longitudinal strain, increased filling pressures [5]), considering the clinical stability on oral diuretics following decongestion and revascularization and the patient preference not to proceed to further invasive management during the current hospitalization, the decision was made to discharge the patient on heart failure medical therapy, with an indication for TAVR re-evaluation in case of further acute decompensation. At last (6-month) follow-up the patient is alive, with clinical stability on medical therapy (NYHA IIb, no angina) and no ensuing cardiac rehospitalizations.

\section{Discussion and conclusions}

ATTR-CA is a common under recognized condition among patients with severe AS, mostly presenting with low-flow low-gradient AS with mildly reduced EF [1]. A high index of suspicion is needed due to the overlapping clinical and echocardiographic presentations of AS patients with and without ATTR-CA. Suspicion should be based on a careful assessment of clinical features including 




Fig. 3 Bone tracer scintigraphy revealing high diffuse myocardial uptake (Perugini score 3)

older age, male sex, bilateral carpal tunnel syndrome, orthostatic hypotension, frequent hospitalizations for heart failure with preserved EF (prior to severe AS diagnosis) and conduction disorders. No association with bicuspid aortic valve disease is reported [6]. Classic echocardiographic signs suggestive of ATTR-CA are frequently inapparent or attributed to severe AS pathophysiology [7]. A relative longitudinal strain apical sparing pattern - while presenting high accuracy for ATTR-CA diagnosis in patients without severe AS - is usually absent in ATTR-CA patients with concomitant severe AS [1]. A tissue Doppler average mitral annular $S^{\prime}$ of $<6 \mathrm{~cm} / \mathrm{s}$ has been recently proposed as a $100 \%$ sensitive measure to screen TAVR candidates for ATTR-CA, with more than half patients with $S^{\prime}<6 \mathrm{~cm} / \mathrm{s}$ having ATTR-CA as confirmed by bone tracer nuclear scintigraphy [1]. Only sparse retrospective evidence is available regarding prognosis of severe AS patients with ATTRCA undergoing aortic valve intervention, mostly limited to surgical replacement and suggesting a high in-hospital and long-term mortality [7].

In this context, no solid evidence is currently available to inform the diagnostic strategies for ATTR-CA among patients with severe AS and, more importantly, whether and in which ATTR-CA patients TAVR (and which with TAVR device [8]) may have a prognostic benefit remains unclear.

In the presented case, a comprehensive heart team evaluation deemed the natural history of ATTR-CA to negatively affect both the patient's prognosis and procedural risk, leading, along with consideration of patient preferences, to a lack of indication to TAVR during the index hospitalization despite severe AS. At mid-term follow-up, the patient was clinically stable without ensuing decompensation episodes, accordingly, clinical follow-up is ongoing. This management strategy, despite based on objective functional, laboratoristic and imaging parameters along with patient preferences, was based on a subjective weight of the numerous elements taken into considerations, as solid data regarding risk stratification and management of severe AS in patients with ATTR$\mathrm{CA}$ are currently lacking. Indeed, no clear guidance to orientate decision making in this setting is available, advocating for prospective studies to clarify if TAVR may have a prognostic benefit in ATTR-CA - and which ATTR-CA-patients.

\section{Abbreviations}

AS: Aortic stenosis; ATTR-CA: Transthyretin cardiac amyloidosis; EF: Ejection fraction; LV: Left ventricle; NYHA: New York heart association;

TAVR: Transcatheter aortic valve replacement

\section{Acknowledgements}

The case was presented as a poster presentation at the $80^{\circ}$ National Congress of the Italian Society of Cardiology (Rome, 12-15 december 2019).

\section{Authors' contributions}

GG, FDA, FB and FL analyzed and interpreted the patient data. AF, AV and AA performed the echocardiographic examination. RC performed the bone tracer scintigraphy exam. FC, GA, MG, PO, FDA and GG were involved in Heart Team discussion. All the authors were major contributor in writing the manuscript. GMDF critically revised the manuscript. The authors approved the manuscript in its current form.

Funding

No funding supported this work. 


\section{Availability of data and materials}

Data sharing is not applicable to this article as no datasets were generated or analysed during the current study.

\section{Ethics approval and consent to participate}

Not applicable.

\section{Consent for publication}

Written informed consent for publication was obtained from the patient.

\section{Competing interests}

The authors declare that they have no competing interests.

\section{Author details}

Department of Medical Sciences, University of Turin, Città della Salute e della Scienza Hospital, Turin, Italy. ${ }^{2}$ Division of Cardiology, Città della Salute e della Scienza, Corso Bramante 88/90, 10126 Turin, Italy.

Received: 5 April 2020 Accepted: 17 May 2020

Published online: 01 June 2020

\section{References}

1. Narotsky DL, Hamid N, Khalique OK, et al. Unveiling transthyretin cardiac amyloidosis and its predictors among elderly patients with severe aortic stenosis undergoing transcatheter aortic valve replacement. Eur Heart J. 2017;38(38):2879-87.

2. Gillmore JD, Maurer MS, Falk RH, et al. Nonbiopsy diagnosis of cardiac transthyretin amyloidosis. Circulation. 2016;133:2404-12.

3. Afilalo J, Lauck S, Kim DH, et al. Frailty in older adults undergoing aortic valve replacement: the FRAILTY-AVR study. J Am Coll Cardiol. 2017;70:689700.

4. Grogan M, Scott CG, Kyle RA, et al. Natural history of wild-type transthyretin cardiac amyloidosis and risk stratification using a novel staging system. J Am Coll Cardiol. 2016;68(10):1014-20.

5. Chacko L, Martone R, Bandera F, et al. Echocardiographic phenotype and prognosis in transthyretin cardiac amyloidosis. Eur Heart J. 2020;41:1439-47.

6. Presbitero $P$, lannetta $L$, Pagnotta $P$, et al. Transcatheter aortic valve implantation in bicuspid anatomy: procedural results with two different types of valves. Minerva Cardioangiol. 2018;66(2):129-35.

7. Ternacle J, Krapf L, Mothy D, et al. Aortic stenosis and cardiac amyloidosis: JACC review topic of the week. J Am Coll Cardiol. 2019;74:2638-51.

8. Hecker F, Arsalan M, Kim WK, Walther T. Transcatheter aortic valve implantation (TAVI) in 2018: recent advances and future development. Minerva Cardioangiol. 2018;66(3):314-28.

\section{Publisher's Note}

Springer Nature remains neutral with regard to jurisdictional claims in published maps and institutional affiliations. 